\title{
SUBOPTIMAL SUPERVISORY LEVEL POWER FLOW CONTROL OF A HYBRID ELECTRIC VEHICLE
}

\author{
Kasemsak Uthaichana*, Sorin Bengea ${ }^{* *}$, Raymond DeCarlo ${ }^{*}$ \\ "Purdue University, School of ECE, Northwestern Ave, West Lafayette, IN, 47907, USA \\ ${ }^{* * *}$ Eaton Innovation Center, 15151 Highway 5 Bldg.2, Eden Prairie, MN, 55344, USA
}

\begin{abstract}
This paper develops a supervisory level power flow suboptimal controller for a hybrid electric vehicle. The power flow model has two modes of operation determined by whether or not the electric motor is motoring or generating. The solution to the hybrid optimal control problem uses the recently developed embedding technique which places the original problem into a parameterized family of problems. The parameterized family of problems, amenable to the application of classical optimal control theory, is then solved. Because of the usual numerical difficulty in solving the state and adjoint equations simultaneously combined with nonlinearities in the model, a suboptimal piecewise solution is obtained for tracking a trapezoidal driving profile. Results are reasonable and encouraging. Copyright $\odot 2005$ IFAC
\end{abstract}

Keywords: HEV; hybrid optimal control; hybrid control; switching systems; supervisory control.

\section{INTRODUCTION}

This paper, building on a companion paper which developed a power flow control model for a HEV, investigates the supervisory level power flow control problem for a parallel configured HEV having an internal combustion engine ICE (diesel) with an electric-motor-battery-pack to propel the vehicle. The model of the companion paper uses a 4-cylinders $1.9 \mathrm{~L}$ diesel engine and a continuously variable transmission (CVT) to potentially enhance the operating efficiency of the ICE. A $57 \mathrm{~kW}$ electric motor (EM) is used in conjunction with a battery pack of twenty-seven of 13 Ah $12 \mathrm{~V}$ lead acid batteries connected in series.

Given a trapezoidal driving profile, the supervisory control must decide how best to utilize the batteryEM and ICE to achieve optimal performance, for example, tracking a driving profile with minimum fuel consumption while maintaining state of charge constraints (SOC) on the battery which entails the timing and extent of time that the electric-motorgenerator EM/GEN is in the generating mode and how best to recover breaking energy. This is termed a Power Management Control Problem. Figure 1 shows a block diagram of the subsystems where arrows indicate potential power flows.

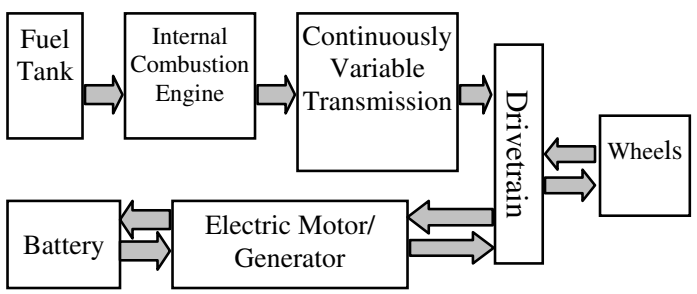

Fig 1. Schematic of HEV power flows.

Once deciding on power flows, the supervisory control then instructs local controllers to implement its computed optimal power flow profiles for each subsystem through a coordinator level. Fig. 2 shows the supervisory level, the coordination level, and the 
local subsystem controllers. Details of the coordinated decentralized control problem and its solution are the subject of a future paper.

The control of an HEV to achieve optimal performance (minimum emissions, maximum fuel economy, satisfaction of drivability constraints in the presence of known/unknown road conditions and driver demands) includes the classical continuous/discrete variables in concert with logical or decision variables that determine the mode of operation. A unified mathematical tractable formulation for the optimal control of such problems has only recently been developed (Bengea and DeCarlo, 2005).

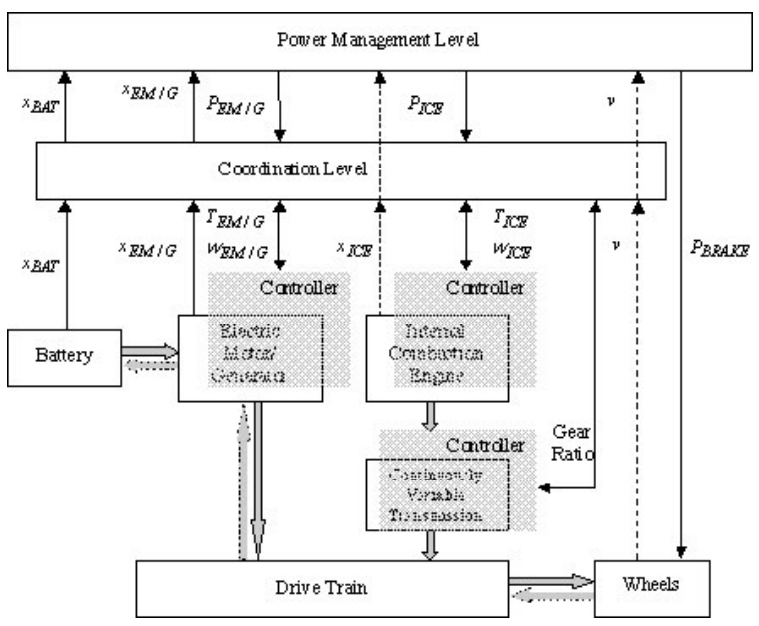

Fig. 2. Supervisory power flow control strategy.

The idea of a supervisory level power flow control problem has occurred earlier in Brahma, et al., (2000) and C.C. Lin, et al., (2003) who adopt a two-level (supervisory and local) hierarchical approach to solving the HEV control problem; their approach uses instantaneous power flow levels, the corresponding efficiencies and/or losses of each subsystem, and the battery SOC in dynamic programming. The conceptual approach taken in this work has elements from both. Yet, in contrast to C.C. Lin, et al., (2003), and whose work used dynamic programming to optimize the fuel economy and emission reduction at the supervisory level, this study strictly solves "power management problem" for the optimal power flow at the supervisory level using vehicle velocity dependent efficiencies. Further, in contrast to Brahma, et al. (2000), the power flow model is fully dynamic containing diff. eqs. in engine power, in vehicle velocity, and in SOC, along with velocity dependent efficiencies which add another level of difficulty to the problem. For other related work on HEV modeling and control strategies, see Powell, et al. (1998); Paganelli, et al., (2001), Phillips, et al.(2000); Saeks, et al. (2002).

According to C.C. Lin, et al., (2003), there are five possible modes of operation for the HEV: motor only, engine only, motor assisted engine, engine charging the battery, and regenerative braking. From the power management perspective of this study, however, there are only two modes of operation: EM/GEN motoring $(v=0)$ and EM/GEN generating $(v=1)$. Situations in which the ICE is off or the EM is off are viewed as 0-valued controls rather than modes of operation. Notice that the vehicle can be motoring while in the GEN mode also.

\section{HEV MODEL}

From the companion modeling paper (Uthaichana et al. 2005), the modeling equations are:

$$
\left[\begin{array}{l}
\dot{P}_{I C E} \\
\dot{\bar{W}}_{b a t} \\
\dot{V}
\end{array}\right]=f_{v(t)}\left(\left[P_{I C E}, \bar{W}_{b a t}, V,\right]^{\mathrm{T}}, u_{v(t)}(t)\right)
$$

where

$$
\begin{aligned}
\dot{P}_{I C E}= & -\frac{1}{\tau_{I C E}} P_{I C E} \\
& +\frac{1}{\tau_{I C E}} P_{I C E}^{\max }\left(\omega_{I C E}\right) u_{I C E}(t) \operatorname{eng}(t) \\
\dot{\bar{W}}_{b a t} \quad & =\frac{d_{3, v}}{W_{b a t}^{\max }} P_{b a t, n o m}^{2}-\left[\ln \left(d_{1, v} \bar{W}_{b a t}+d_{2, v}\right)\right. \\
& \left.+2 d_{3, v} P_{b a t, n o m}+d_{4, v}\right] \frac{P_{b a t}^{\max }}{W_{b a t}^{\max }} u_{b a t}(t)
\end{aligned}
$$

with, $d_{1,0}=-0.2856, d_{2,0}=1.4734, d_{3,0}=$

$$
\begin{gathered}
7.569 \cdot 10^{-3}, d_{4,0}=0.6834, d_{1,1}=-0.2384, d_{2,1} \\
=1.4852, d_{3,1}=6.872 \cdot 10^{-3}, d_{4,1}=0.6635, \text { and } \\
\dot{V}=-\left(\frac{k_{v 1}}{m_{c}} V^{2}+k_{v 2}\right) \operatorname{sgn}(V)-\frac{1000}{m_{c}\left(V+\varepsilon_{V}\right)} P_{b}(t) \\
+\frac{1000 \varepsilon_{d} \varepsilon_{c}}{m_{c}\left(V+\varepsilon_{V}\right)} P_{F}(t)
\end{gathered}
$$

(Note: the first term in equation $2 \mathrm{c}$ is aerodynamic drag and rolling resistance losses.) When motoring $v(t)=0, P_{F}(t)=P_{C}(t), P_{b}(t)=P_{\text {brake }}^{\max }(t) \cdot u_{\text {brake }}(t)$, $u_{0}=\left[u_{I C E}(t), \text { eng }(t), u_{\text {bat }}(t), u_{\text {brake }}(t)\right]^{\mathrm{T}}, P_{\text {bat }, \text { nom }}=$; $17 \mathrm{~kW}$, when generating $v(t)=1$, $u_{1}=\left[u_{I C E}(t), \operatorname{eng}(t), u_{b a t}(t), u_{\text {brake }}(t), \operatorname{split}(t), r(t)\right]^{T}$, $P_{b}(t)=\frac{1}{\varepsilon_{d}} P_{r e g}^{\max }(t) r(t)+P_{\text {brake }}^{\max }(t) u_{\text {brake }}(t), P_{F}(t)=$ $(1-\operatorname{split}(t)) \eta_{t r} P_{I C E}(t) \operatorname{eng}(t), P_{b a t, n o m}=-18 \mathrm{~kW}$ where (i) $P_{I C E}$ is ICE power in $\mathrm{kW}$ with $P_{\mathrm{ICE}}^{\max }$ being the maximum; while (ii) $\bar{W}_{b a t}$ is the normalized battery energy or SOC while $W_{b a t}^{\max }$ is the 
maximum battery energy, (iii) $V$ is the vehicle velocity, (iv) $u_{I C E}(t)$ is the normalized ICE power control input (v) eng $(t) \in\{0,1\}$ denotes off-on operation of the ICE, (vi) $u_{b a t}(t)$ is the normalized battery power control input, (vii) $u_{\text {brake }}(t)$ is the normalized control while $P_{\text {brake }}^{\max }(V)$ is the maximum mechanical braking power, (viii) split $(t)$ is the normalized fraction of ICE power diverted to the generator, (ix) $r(t)$ is the normalized fraction of the maximum regenerative braking power, (x) $\tau_{I C E}$ is the nominal ICE delay; (xi) $P_{F}(t)$ is the propelling power, (xii) $P_{b}(t)$ is the total braking power, (xiii) $\varepsilon_{d}$ is differential efficiency, (xiv) $\varepsilon_{V}$ is a regularization term.

\section{POWER MANGEMENT CONTROL OF HEV}

In this section we address the power management control problem at the supervisory level. The goal is to compute the desired power level command profiles for the main subsystems of the HEV that achieve a balance between power consumption and reasonable velocity tracking of an a priori given desired velocity profile-dashed trapezoidal curve in fig. 5 specified over the time interval $[0,12] \mathrm{s}$ having the analytical expression,

$$
\begin{aligned}
& V^{\text {des }}=\frac{14}{6} t \cdot 1^{+}(t)-\frac{14}{6}(t-6) \cdot 1^{+}(t-6) \\
& -\frac{14}{3}(t-9) \cdot 1^{+}(t-9)+\frac{14}{3}(t-12) \cdot 1^{+}(t-12)
\end{aligned}
$$

where $1^{+}(t)$ denotes the step function. The control objective is achieved by a performance measure chosen so that both power consumptions and vehicle velocity tracking error are penalized. The following subsection formulates rigorously the optimal control problem.

\subsection{Optimal Control Problem Formulation}

In the motoring mode the important controls goals considered here are:

(i) Minimize $\left[V(t)-V^{d e s}(t)\right]^{2}$ to achieve tracking of $V^{\text {des }}$, equation 3;

(ii) Minimize ICE power usage so as to minimize fuel consumption;

(iii) Operate ICE in regions of high torque-speed efficiency, i.e., to operate the ICE at velocities for which the ICE efficiency $\eta_{I C E}$ is large;

(iv) Maintain the (normalized) energy of the battery (SOC) within a prescribed range, [0.4, 0.8]; hence, we penalize the deviations of SOC from the nominal value of 0.6 ;

(v) Minimize battery power usage.
These goals suggest the following cost function for the motoring $(v(t)=0)$ and generating $(v(t)=1)$ modes:

$$
\begin{gathered}
J_{v(t)}=\int_{t_{0}}^{t_{f}} f_{v(t)}^{0}\left(\left[P_{I C E}, \bar{W}_{b a t}, V\right]^{\mathrm{T}}, u_{v(t)}(t)\right) d t \\
=\int_{t_{0}}^{t_{f}}\left(\kappa_{1, v}\left[V-V^{d e s}\right]^{2}+\kappa_{2, v}\left[P_{I C E}^{\max } u_{I C E}(t)\right]^{2} \operatorname{eng}(t)\right. \\
+\left(\kappa_{3, v}\left[\frac{P_{I C E}}{\eta_{I C E}\left(P_{I C E}, V\right)}\right]^{2}+\kappa_{4, v}\left[\frac{P_{I C E}\left(V-V^{\text {des }}\right)}{\eta_{I C E}\left(P_{I C E}, V\right)}\right]\right) \text { eng }(t) \\
+\kappa_{5, v}(1-v)\left[P_{b a t}^{\max } u_{b a t}(t)\right]^{2}+\kappa_{6, v}\left[\bar{W}_{b a t}-\bar{W}_{b a t}^{\text {avg }}\right]^{2} \\
\left.+\kappa_{7, v}(v)\left[P_{G E N}\right]^{2}\right) d t
\end{gathered}
$$

where $\kappa_{1, v}=\bar{C}_{V}^{v}=2000 / V_{\max }^{2}=10.204 \quad, \quad \kappa_{2, v}=$ $\bar{C}_{I C E, a}^{v}=4 / \max \left(P_{I C E}^{\max }\right)^{2}=7.48 \cdot 10^{-4} \quad, \quad \kappa_{3}=$ $\bar{C}_{I C E, b}^{v}=0.1 / \max \left(P_{I C E}^{\max } / \eta_{I C E}\right)^{2}=7.48 \cdot 10^{-7}$, $\kappa_{4, v}=\bar{C}_{V, I C E}^{v}=-\sqrt{\bar{C}_{V}^{v}} \sqrt{\bar{C}_{I C E, b}^{v}} \quad=-2.76 \cdot 10^{-3}$, $\kappa_{5,0}=\bar{C}_{P b a t}^{0}=500 /\left(P_{b a t}^{\max }\right)^{2}=0.05 \quad, \quad \kappa_{6, v}=\bar{C}_{b a t}^{v}$ $=1000$, and $\kappa_{7, v}=\bar{C}_{G E N}^{1}=(0.17)^{2} /\left(0.17 P_{G E N}^{\max }\right)^{2}$ $=1 /\left(P_{G E N}^{\max }\right)^{2}=3.078 \cdot 10^{-4}, \quad\left(0.17 P_{G E N}\right.$ is the average loss due to battery charging via the generating power) are normalized coefficients for the cost function. The normalized coefficients are chosen to ease the analysis and interpretation of each term in the cost function; moreover, adjusting the importance of each term in the cost function can be done more intelligently on the normalized scale where relative weighting is important.

Hence the hybrid optimal control objective in the embedded framework, $v(t) \in[0,1]$, of Bengea and DeCarlo (2005) is:

$$
\min _{v, u_{v}} \int_{t_{0}}^{t_{f}} f_{v}^{0}\left(\left[P_{I C E}, \bar{W}_{b a t}, V\right]^{\mathrm{T}}, u_{v}(t)\right) d t
$$

subject to the set of dynamical equations (1) and (2) and that the control inputs are contained in the unit interval.

\subsection{Optimal Control Input Construction}

The optimal control algorithm, solved at the supervisory level, is based on the theoretical procedure developed in Bengea and DeCarlo (2005) for switching systems. The proposed algorithm computes the optimal control inputs as well as the operating mode by minimizing the objective function (5) subject to the state dynamical constrained (1) and 
(2). The state constraints are enforced by the use of the Lagrange multiplier $\lambda=\left[\lambda_{1}, \lambda_{2}, \lambda_{3}\right]^{T}$. In the absence of a final terminal state constraint, we take, as usual, without loss of generality, the Lagrange multiplier of the performance index, $\lambda_{0}=-1$. The procedure for determining the suboptimal control inputs consists of the following steps at each time t:

Step 1: Compute the Hamiltonians for each mode of operation

Step 1a: Define the Hamiltonian $H_{0}$ associated with the motoring mode as:

$$
H_{0}=\lambda_{0} \cdot f_{0}^{0}+\left[\lambda_{1}, \lambda_{2}, \lambda_{3}\right] \cdot f_{0}
$$

Step 1b: Define the Hamiltonian $H_{1}$ associated with the generating mode as:

$$
H_{1}=\lambda_{0} \cdot f_{1}^{0}+\left[\lambda_{1}, \lambda_{2}, \lambda_{3}\right] \cdot f_{1}
$$

Step 2: Compute the control inputs for each mode of operation as the arguments that maximize the Hamiltonians; determine the optimal mode of operation

Step 2a: Compute

$$
u_{0}(t)=\left[u_{I C E}(t), \operatorname{eng}(t), u_{\text {bat }}(t), u_{\text {brake }}(t)\right]^{T}
$$

such that $H_{0}$ is maximized i.e.,

$$
u_{0}(t)=\arg \max \left\{\lambda_{0} f_{0}^{0}+\lambda^{T} f_{0}\right\}
$$

Step 2b: Compute $u_{1}=\left[u_{I C E}(t)\right.$, eng $(t), u_{b a t}(t)$,

$\left.u_{\text {brake }}(t), \operatorname{split}(t), r(t)\right]^{T}$, such that $H_{1}$ is maximized i.e.,

$u_{1}(t)=\arg \max \left\{\lambda_{0} f_{1}^{0}+\lambda^{T} f_{1}\right\}$

Step 2c: $v=\arg \max \left\{H_{0}, H_{1}\right\}$ with the

Hamiltonians evaluated at the control inputs from steps $2 \mathrm{a}$ and $2 \mathrm{~b}$, respectively.

Step 3: Determine the derivatives of the state and the adjoint state

Step 3a: If $v=0$ then $\dot{x}(t)=f_{0}\left(x(t), u_{0}(t)\right)$ and

$$
\dot{\lambda}(t)=-\lambda_{0}\left[\frac{\partial f_{0}^{0}}{\partial x}\right]-\left[\frac{\partial f_{0}}{\partial x}\right]^{T} \lambda(t)
$$

where the partial derivatives are evaluated at the state $x(t)$, the adjoint state, $\lambda(t)$, and the control input, $u_{0}(t)$.

Step 3b: If $v=1$ then

$$
\begin{aligned}
& \dot{x}(t)=f_{1}\left(x(t), u_{1}(t)\right) \\
& \dot{\lambda}(t)=-\lambda_{0}\left[\frac{\partial f_{1}^{0}}{\partial x}\right]-\left[\frac{\partial f_{1}}{\partial x}\right]^{T} \lambda(t)
\end{aligned}
$$

where the partial derivatives are evaluated at the state $x(t)$, the adjoint state $\lambda(t)$ and the control input $u_{1}(t)$.

Although the above equations represent necessary conditions for optimality, the proposed control cannot be immediately derived due to the unknown value for the initial adjoint state $\lambda(0)=\left[\lambda_{1}(0), \lambda_{2}(0), \lambda_{3}(0)\right]^{T}$. Moreover, the adjoint state must satisfy the boundary constraint $\lambda\left(t_{f}\right)=0^{T}$, a consequence of unconstrained terminal state . Further with the difficulties given by the HEV nonlinear equations and by the switching behavior, there results challenging numerical problems.

\subsection{Suboptimal Control Input Construction}

To overcome the difficulties mentioned in the previous section, we divide the optimization interval into three subintervals and solve three separate optimization problems:

$$
\min \int_{t_{0}^{i}}^{t_{f}^{i}} f_{v(t)}^{0}\left(\left[P_{I C E}, \bar{W}_{b a t}, V, u_{v(t)}(t)\right]^{\mathrm{T}}\right) d t
$$

for $\left[t_{0}^{i}, t_{f}^{i}\right]=\{[0,6],[6,9],[9,12]\}, \mathrm{i}=1,2,3$, subject to a continuous state over the entire interval, i.e., for a feasible suboptimal solution, we require $x\left(t_{f}^{i}\right)=x\left(t_{0}^{i+1}\right)$. For an overall optimal solution, $\lambda\left(t_{f}^{3}=12\right)=0^{T}$. Hence we solve for the suboptimal solution forward and backward in time as follows: First, we minimize the cost function (equ 10) over the interval $[9,12] \mathrm{s}$ subject to $\lambda\left(t_{f}^{3}\right)=0^{T}$ and a desired $x\left(t_{f}^{3}\right)=[0,0.6,0]^{T} \cong\left[2.45 \cdot 10^{-6}, 0.6,0.04\right]^{T}$.

This approximate terminal condition is chosen for numerical reasons. The obtained cost, and control input are optimal over $[9,12] \mathrm{s}$ with the initial condition $x\left(t_{0}^{3}\right)$ that results from the backward simulation. Second, we consider the interval $[0,6] \mathrm{s}$ for which we have a known initial condition $x\left(t_{0}^{1}\right)=x(0)=[0,0.6,0]^{T}$. We then guess or search for the proper adjoint state initial condition, $\lambda(0)$ to produce a state $x\left(t_{f}^{1}\right)$ within an acceptable range of performance: $P_{I C E}(t=6) \in[40,73] \mathrm{kW}, \bar{W}_{b a t}(t=6)$ $\in[0.55,0.6]$ where from simulation studies the lower bound is sufficient for the EM to operate at its full capacity for the first six seconds, and $V(t=6) \in[12.5,15] \mathrm{m} / \mathrm{s}$. Then we search within a small ball about the nominal value of $\lambda(0)$ that produced an acceptable $x\left(t_{f}^{1}\right)$ to produce a locally minimal cost function. We then set $x\left(t_{0}^{2}\right)=x\left(t_{f}^{1}\right)$ and $x\left(t_{f}^{2}\right)=x\left(t_{0}^{3}\right)$ and minimize the cost function (equ. 10) by finding consistent values of the adjoint variable terminal conditions over [6,9] s. The simulation results both forward and backward in time are obtained via the usage of ode15s function solver in MATLAB with the options of error tolerance of $10^{-4}$ for a scalar, and error tolerance of $10^{-8}$ for each 
component of a vector. The discussion and presentation on the numerical results are given in the next sections.

\section{SIMULATION RESULTS}

In this section we present the results of the suboptimal control developed as per the description in the previous section. The coefficients in the cost function, equ (4), are selected such that $\bar{C}_{b a t}^{v}>C_{V}^{v}>\bar{C}_{I C E, a}^{v}>\bar{C}_{I C E, b}^{v}$ i.e., the deviation of $\bar{W}_{b a t}$ from $\bar{W}_{a v g}=0.6$ is more heavily weighted than the vehicle velocity tracking error, which is considered more important than ICE power usage. Control inputs were computed for three separate intervals, $[9,12],[0,6]$ and $[9,6] \mathrm{s}$, respectively. The simulation results for the three intervals are combined and plotted in Figs. 3-6.

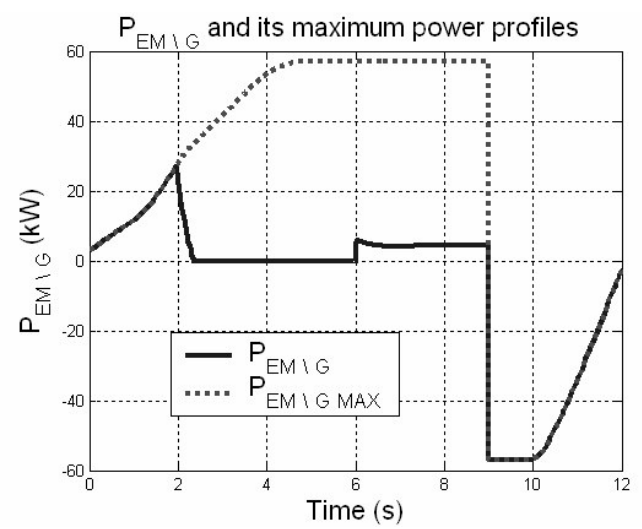

Fig 3. EM/GEN power absorption/delivery with superimposed plot of $P_{E M / G E N}^{\max }$.

At startup, the EM (fig 3) operates at its maximum (speed dependent) power level to propel the vehicle. There is insufficient available power for perfect tracking. At $V>1.877 \mathrm{~m} / \mathrm{s}$ (around $t=1.1 \mathrm{~s}$ ), the ICE kicks in, while EM continues to deliver maximum (speed dependent) power or equivalently absorbs maximum allowable power from the battery up to $t=2 \mathrm{~s}$. The mechanical powers from both sources, at their full or almost full capacities, are delivered to the driveshaft, to reduce the tracking error over $[1.1,2] \mathrm{s}$. Note that ICE delivers its power during vehicle acceleration at a level slightly below its maximum steady-state level due to the delay $\tau_{\text {ICE }}$ in the ICE dynamics as per Fig. 5.

At $2 \mathrm{~s}$ when $V$ approximates the desired speed, the EM reduces its power to zero by $t=3 \mathrm{~s}$. Over the interval $[4,6] \mathrm{s}$, the ICE operates at its maximum allowable power, without assistance by the EM. Perfect tracking is not attained because the penalty coefficients for battery power usage and/or deviation of the SOC from nominal are overriding the penalty for imperfect tracking over this interval.

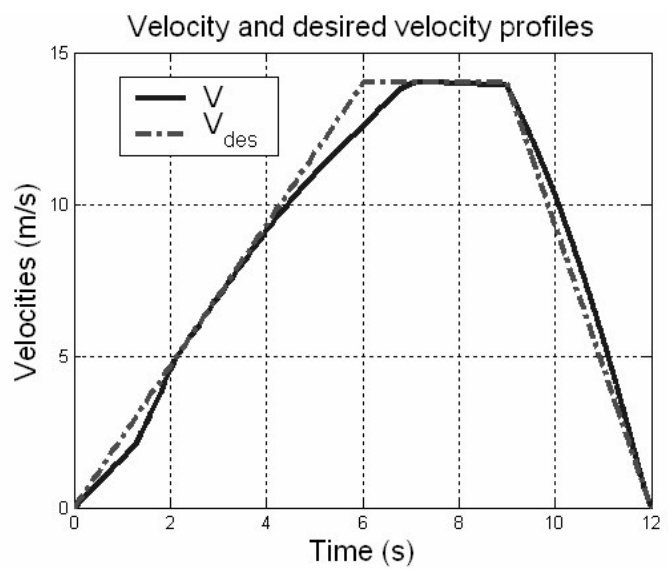

Fig. 4. HEV's Velocity during $[0,12] \mathrm{sec}$ on with superimposed desired velocity profile.

Nevertheless the EM kicks in at about $6 \mathrm{~s}$ and in concert with the ICE, drives the vehicle to track the desired profile.

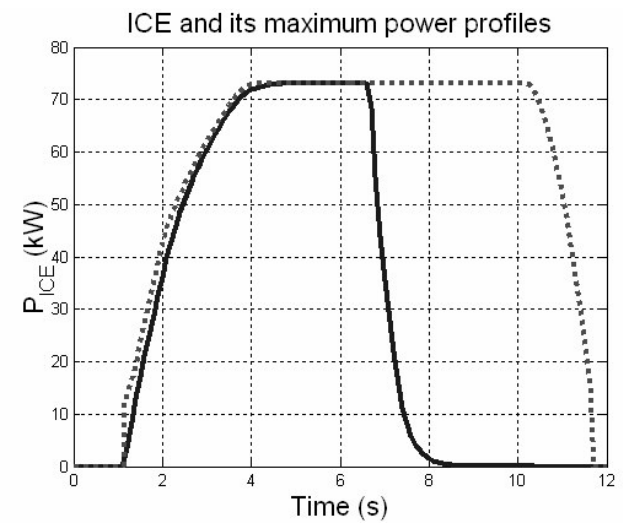

Fig. 5. ICE Power usage during $[0,12] \mathrm{sec}$ with superimposed $P_{I C E}^{\max }$.

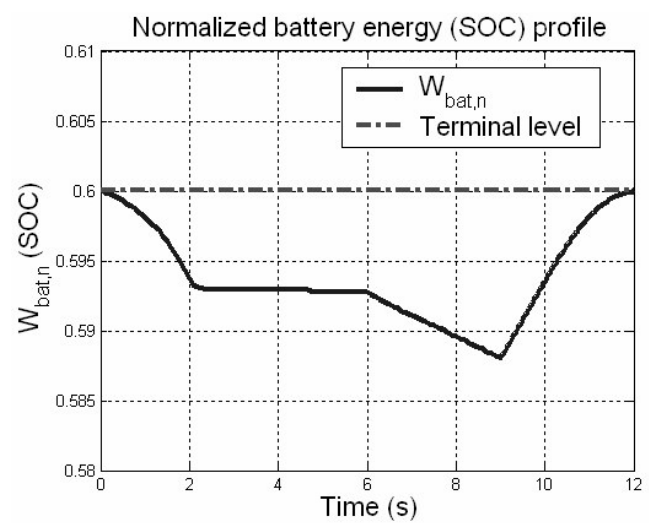

Fig 6. Battery energy profile during $[0,12] \mathrm{sec}$.

However, as the vehicle approaches perfect tracking, eliminating further acceleration, the ICE kicks out while the EM maintains the velocity tracking over [7,9] s. Here, the power required to sustain the vehicle speed is less than $20 \mathrm{~kW}$. Hence, only the EM needs to provide power.

Finally, during the last 3 seconds, regenerative braking is engaged to recover a portion of vehicle 
kinetic energy to replenish battery power and increase its SOC back its starting level of 0.6

\section{CONCLUSION}

A supervisory level power flow suboptimal controller for a hybrid electric vehicle is developed and applied to the HEV dynamical model developed in Uthaichana, et al. (2005). The controller development is based on a solution to the hybrid optimal control problem (Bengea and DeCarlo, 2005) which uses an embedding technique to place the existing problem into a parameterized family of problems. The parameterized family of problems is amenable to the application of classical optimal control theory. The resulting state-adjoint equations arising from the associated Hamiltonian are numerically sensitive and stiff. Hence, they are solved over three separate intervals to achieve a suboptimal solution for which the state vector is continuous. Further work on numerical solution of the equations is needed, but this of course is an age old problem.

Based on the supervisory level power flow model, this paper sets forth a suboptimal solution to this problem that demonstrates reasonable drivability and power usage. Local subsystem controllers must now be developed to track these profiles. Hence, the HEV control problem is framed as a Hierarchical problem so as to separate the power flow problem from the development of local controllers to achieve these profiles. Coordination of such decentralized subsystem controllers is a topic of future research. In addition, experimentation with different cost function weights will lead to different performance. It is expected that future work will look at allowing a larger deviation in battery state of charge to permit better vehicle tracking. Further it would be interesting to evaluate the control strategy for more complicated driving profiles.

\section{ACKNOWLEDGEMENT}

The seeds for motivating this work were planted by the Center for Automotive Research at The Ohio State University during the spring of 2002. This work was not supported by NSF or any government agency.

\section{REFERENCES}

S. Bengea and R. DeCarlo (2005), "Optimal Control of Switched/Hybrid Systems with Applications to the control of Hybrid Electric Vehicles," Automatica, vol. 41, Jan. 2005, pp. 11-27.

A. Brahma, Y. Guezennec and G. Rizzoni (2000), "Dynamic Optimization

of Mechanical/Electrical Power Flow in Parallel Hybrid Electric Vehicles," in Proc. AVEC the, $5^{\text {th }}$ International Symposium on Advanced Vehicle Control, Ann Arbor, MI, Aug.
C.C. Lin, H. Peng, J. Grizzle and J. Kang (2003), "Power Management Strategy for a Parallel Hybrid Electric Truck," IEEE Transactions on Control Systems Technology, 11(6), pp. 839-849.

G. Paganelli, M. Tateno, A. Brahma, G. Rizzoni, and Y. Guezennec (2001), "Control development for a hybrid-electric sport-utility vehicle: strategy, implementation and field test results. in IEEE Proc. of American Control Conference, Arlington, VA, pp. 5064-5059.

A. Phillips, M. Jankovic, K. Bailey, (2000), "Vehicle system controller design for a hybrid electric vehicle," in Proc of the 2000. IEEE Int Conf on Control App, Anchorage, AK, USA.

B. Powell, K. Bailey, and S. Cikanek (1998), "Dynamic modeling and control of hybrid electric vehicle powertrain systems," IEEE Control Systems Magazine, 18(5), pp 17-22.

R. Saeks, C. Cox, J. Neidhoefer, P. Mays and J. Murray (2002), "Adaptive control of a hybrid electric vehicle," IEEE Transactions on Intelligent Transportation Systems, 3(4), pp. 213-234.

K. Uthaichana, S. Bengea and R. DeCarlo (2005), "HEV modeling for a supervisory level power flow control problem," in Proc of 2005 IFAC World Congress, Prague. 